\title{
NGHIÊN CÚU VECTOR TILES VÀ FRAMEWORK NODEJS TRONG XÂY DỤ̂NG MÁY CHỦ CUNG CẤP BẢN ĐỒ DẠNG TILE
}

\author{
NGUYẼ̃N HOÀNG LONG ${ }^{(1)}$, ĐINH BẢO NGỌC(1), TRÀN THỊ HẢI VÂN ${ }^{(1)}$

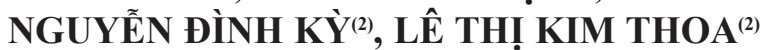 \\ (1)Truòng Đại học Mỏ-Địa chất \\ (2) Viện Hàn lâm Khoa học và Công nghệ Việt Nam
}

\section{Tóm tắt:}

Việc tối ư hóa khả năng trục quan hóa và tăng khả năng truy vấn dũ liệu không gian trên WebGIS là yếu tố cần được quan tâm khi phát triển ứng dụng WebGIS. Kĩ thuật mảnh bản đồ (tiled map) đặc biệt là định dạng mảnh ảnh (raster tiles) đã được giới thiệu và cung cấp bởi nhiều hệ thống máy chủ GIS nhu ArcGIS Server, GeoServer với mục đích tăng tốc độ hiển thị dũ liệu không gian. Tuy nhiên, raster tiles có nhược điểm là dũ liệu được gửi tới máy khách chi duới dạng hình ảnh. Điều này dẫn đến hạn chế trong việc truy vấn dũ liệu. Ngoài ra, việc định dạng các kiểu hiển thị cho các đối tượng trên bản đồ cũng bị hạn chế. Kĩ thuật mói vector tiles đã ra đời nhằm khắc phuc các hạn chế của raster tiles. Bài báo này trình bày tổng quan về kỹ thuật tiles map đặc biệt là dịnh dạng vector tiles. Ngoài ra, bài báo cũng trình bày các bước trong việc xây dựng một úng dụng cung cấp dịch vu vector tiles dụa trên các nền tảng mã nguồn mỏ miễn phí là: PostgreSQL, NodeJS và MapNik

\section{Mở đầu}

Úng dụng web GIS đầu tiên được xây dựng bởi Xerox vào năm 1993. Ứng dụng này thể hiện bản đồ dưới dạng một tấm ảnh, khi người dùng di chuyển đến một phạm vi khác trên bản đồ, một tấm ảnh mới sẽ được tải về để hiển thị vị trí đó. Phương pháp này có nhược điểm là làm chậm tốc độ của trang web, làm giảm trải nghiệm người dùng. Đề khắc phục điều này, vào năm 2005 Google đưa ra giải pháp hiển thị bản đồ mới bằng việc chia nhỏ bản đồ thành các tấm ảnh nhỏ, có liên kết với nhau. Phương pháp này được biết đến với tên gọi raster tiles [1] và vẫn còn được phổ biến cho tới ngày nay.

Phương pháp raster tiles cho đến nay vẫn không có sự thay đổi nhiều về kĩ thuật. Raster tiles sẽ chia bản đồ thành các ô và định dạng các kiểu thể hiện bản đồ sẵn và được thể hiển sẵn trên hệ thống máy chủ. Định dạng bản đồ giống nhau đối với tất cả người dùng. Tuy nhiên với raster tiles việc truy xuất thông tin đối tượng trên bản đồ gặp nhiều hạn chế. Để khắc phục điều này, các nhà nghiên cứu đã đưa ra phương pháp vector tiles [2]. Vector tiles cho phép chia nhỏ dữ liệu vector thành các ô và định dạng từng ô sẽ được biểu ở phía máy khách. Điều này cho phép đa dạng hóa khả năng biểu diễn các đối tượng bản đồ như là cho phép người dùng thay đổi biểu tượng các đối tượng trên bản đồ. Ngoài ra, việc truyền dữ liệu bản đồ dạng vector sẽ làm việc hiển thị bản đồ được trơn mượt không bị vỡ hình. Một ưu điểm khác là việc tương tác và truy vấn với các đối tượng trên bản đồ được cải thiện đáng kể.

\section{Tổng quan về kĩ thuật tiles map}

Với các ứng dụng web GIS ngày trước, bản đồ sẽ được tải toàn bộ về phía trình duyệt của người dùng dưới dạng một tấm ảnh có dung lượng rất lớn, khi người dùng thực hiện các thao tác với bản đồ như phóng to, thu nhỏ, dịch chuyển bản đồ thì tấm ảnh đó sẽ được tải lại. Vấn đề phát sinh là tấm ảnh có dung lượng lớn nếu máy tính người dùng có cấu hình không cao hoặc đường truyền internet chậm thì việc tải bản đồ sẽ 
mất nhiều thời gian, ảnh hưởng tới năng suất làm việc và trải nghiệm của người dùng.

Bản đồ Google map ra đời năm 2005 đi cùng với một phương pháp có tên là tiled đã thay đồi đáng kể tốc độ hiển thị và thao tác với bản đồ số thông qua môi trường mạng internet. Thay vì tải toàn bộ bản đồ dưới dạng một tấm ảnh lớn, Google đã chia bản đồ thành các ô vuông nhỏ dưới dạng lưới với các kích thước xác định. Do có cấu trúc dạng lưới, các mảnh bản đồ sẽ được hiển thị theo vị trí người dùng muốn xem thay vì phải tải toàn bộ bản đồ. Theo cách chia các tấm ảnh bản đồ của google mỗi tấm ảnh sẽ có kích cõ̃ $256 * 256$ điểm ảnh.

Độ chi tiết của bản đồ phụ thuộc vào kích thước của tấm ảnh. Việc thêm chi tiết của bản đồ sẽ phải tăng kích thước của ảnh Việc làm này khá dễ dàng với các bản đồ chuyên đề, biết trước mục đích sử dụng và tỷ lệ bản đồ. Tuy nhiên với bản đồ sử dụng chung cho nhiều mục đích khác nhau (ví dụ như các bản đồ nền gồm 7 lớp thông tin) khi phải tải lại trang web sẽ gây mất thời gian do dung lượng của bản đồ nền cần tải về lớn

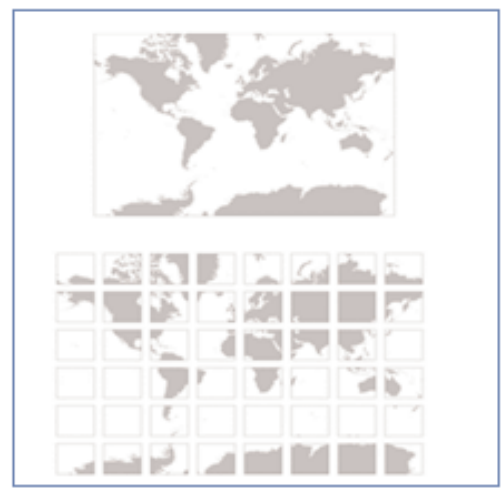

Hinh 1: Tiled map

Một tấm ảnh google map bao trùm toàn bộ thế giới có kích thước 256 * 256 điểm ảnh tuy nhiên ở mức phóng ảnh 20 lần thì kích thước của bức ảnh sẽ tăng lên 134 triệu * 134 triệu điểm ảnh. Kích thươc lưu trữ tấm ảnh này lên tới 25 terrabytes, không phù hợp với bất kì cấu hình máy tính người dùng nào.

Công thức tính tính tọa độ pixel như sau: tọa độ điểm ảnh= tọa độ thực $* 2^{\text {múc phóng }}$

Từ công thức trên cho thấy ở mỗi mức phóng sẽ tăng gấp đôi cả tọa độ $\mathrm{x}$ và $\mathrm{y}$. Do đó, mỗi mức thu phóng cao hơn sẽ tăng độ phân giải lên 4 lần. Ví dụ ở mức thu phóng 1 sẽ bao gồm 4 ô độ phân giải 256 * 256 điểm ảnh. Kết quả toàn bộ bản đồ sẽ có kích thước $512 * 512$ điểm ảnh. Ở mức thu phóng 19 theo tọa độ $\mathrm{x}$ và $\mathrm{y}$ có thể được tham chiếu theo khoảng giá trị từ 256 * 219. Ở mức thu phóng 0 tọa độ pixel bằng tọa độ thực (tọa độ thế giới).

Để biểu thị chính xác từng vị trí trên bản đồ, ở mỗi mức thu phóng các JavaScript API sẽ đưa ra khung xem bản đồ dưới dạng tọa độ vĩ độ và kinh độ thành các tọa độ điểm ảnh. Sau đó API sẽ các định các mảnh đồ đồ nằm trong giới hạn khung xem. Mỗi mảnh bản đồ sẽ được đại diện tham chiếu bởi tọa độ của từng mảnh được lưu trữ trong cơ sở dữ liệu.

Tọa độ của các mảnh bản đồ (tilesets) sẽ có tọa độ $\mathrm{x}$ tăng dần theo chiều từ tây sang đông và tọa độ $\mathrm{y}$ tăng dần theo chiều từ bắc xuống nam [3].

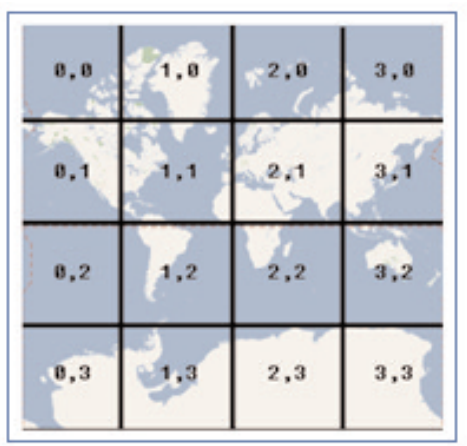

Hình 2: Cách xác định tọa độ của tùng mảnh

Số mảnh tile được tính theo công thức sau: số mảnh $=2 \mathrm{z} * 2 \mathrm{z}$. Trong đó $\mathrm{z}$ là tỷ lệ mức phóng. Áp dụng công thức này ta thấy: ở mức mức phóng $=2$ số tiles là 16 , ở mức mức phóng $=3$ số tiles là 64 mảnh 


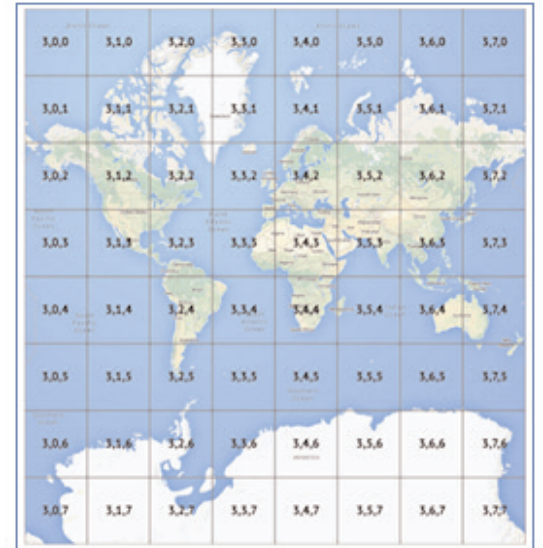

Hình 3: Số lượng tile ở mưc mức phóng $=3$

\subsection{Phân loại bản đồ tile}

Hiện nay có 2 định dạng mảnh (tiles) được sử dụng là Raster tiles và Vector tiles. Trong đó Raster tiles được sử dụng từ lâu còn Vector tiles mới được sử dụng trong thời gian gần đây. Các nhà cung cấp bản đồ như Google, Esri cung cấp các bản đồ nền thông qua các bản đồ đa mức thu phóng. Bản đồ sẽ được chuyển đổi sang các định dạng ảnh như PNG hay JPG và được tối ưu về mặt hiệu năng. Tuy nhiên các bản đồ này có các hạn chế như: người dùng không thể tùy biến bản đồ, các tệp ảnh bản đồ không tùy biến với độ phân giải màn hình [4].

Nhằm cung cấp thêm sự lựa chọn cho người sử dụng, các nhà cung cấp dịch vụ bản đồ đã đưa ra một lựa chọn mới đó là bản đồ nền dạng vector. Bản đồ nền dạng vector dựa trên nền tảng các vector tiles (định dạng PBF) được chuyển đổi ở phía client-side đi kèm với một tệp quy định kiểu.

Raster tiles chia bản đồ thành các mảnh và chuyển đổi bản đồ thành các tệp dạng ảnh được chuẩn bị sẵn ở phía server ở các định dạng như JPG hay PNG. Raster tiles được cung cấp đến người dùng thông qua các dịch vụ web như WMS (Web Map Service) hay WMTS (Web Map Tiles Service). Raster tiles có các ưu điểm sau:

+ Làm việc tốt trên tất cả các ứng dụng và thiết bị bao gồm các ứng dụng máy tính để bàn, các ứng dụng trên điện thoại di động hay trên các trình duyệt Web.

+ Cung cấp khả năng thao tác với các nhà làm bản đồ như dán nhãn hay biểu tượng các đối tượng trên bản đồ.

+ Hỗ trợ tốt các dữ liệu dạng ảnh và cả dữ liệu độ cao.

+ Cung cấp chế độ in ấn trên các trình duyệt web

Vector tiles cũng chia bản đồ thành các mảnh tiles tuy nhiên dữ liệu được lưu dưới dạng vector (dạng điểm, đường, vùng). Mỗi một mảnh vector tiles sẽ bao gồm dữ liệu không gian (hình họcgeometries) và dữ liệu thuộc tính như tên đường, tên địa điểm, số nhà. Vector tiles chỉ được chuyển đổi khi có yêu cầu từ phía người dùng trên trình duyệt. So với Raster tiles, Vector tiles có 2 lợi raster tiles thế như sau [3]:

+ Các thực hiển thị (Styling): giống dữ liệu dạng vector khác, người dùng có thể tùy chỉnh (chọn) các cách thế hiện khác nhau cho các đối tượng trên bản đồ

+ Kích thước (Size): so với raster tiles, dung lượng của Vector tiles nhỏ hơn rất nhiều. Điều này cho phép bản đồ được tải về nhanh chóng và thuận tiện trong việc tăng tốc độ truy xuất dữ liệu và giảm tải cho hệ thống (caching).

Vector tiles được mã hóa bỏi Google Protobufs $(\mathrm{PBF})$. Nó cho phép sắp xếp cấu trúc dữ liệu. Để định dạng cấu trúc của PBF file, dữ liệu sẽ được mã hóa 2 thành phần hình học và thuộc tính. Quá trình mã hóa thông tin hình học sẽ diễn ra như sau: tọa độ của các vector tiles sẽ được chuyển đổi từ hệ tọa độ địa lý (kinh độ hoạc vĩ độ) sang tọa độ dạng lưới. Các vector tiles mã hóa các điểm đường, vùng như là các cặp tọa độ $\mathrm{x}, \mathrm{y}$ có quan hệ tương đối bắt đầu từ trái sang và trên xuống.

\section{Vector tiles}

\subsection{Tổng quan về Vector tiles}

Vector tiles là giải pháp được google đưa ra năm 2010 trên phiên bản di động của ứng dụng 
google map, năm 2013 giải pháp này được áp dụng trên phiên bản website của google map. Vector tiles sẽ lưu trữ các đối tượng vector như điểm, đường, vùng phía máy chủ (server) thay vì các tấm ảnh như raster tiles.

Mô hình vector tiles tương tự như raster tiles là chia dữ liệu dataset thành ô lưới, mỗi ô lưới sẽ chứa và hiển thị dữ liệu dạng vector. Nếu một đối tượng hiển thị trên 2 ô lưới khác nhau, đối tượng đó sẽ bị chia thành các phần hiển thị trên các ô khác nhau. Tương tự như raster tiles, dữ liệu sẽ được chia nhỏ thành các tiles khi có yêu cầu từ phía người dùng. Đa số các ứng dụng vector tiles đều sử dụng Google XYZ schema. Cách xác định tham số của vector tiles hoàn toàn tương tự như raster tiles. Ví dụ: mức độ thu phóng sô 9 , cột 8 dòng 4 sẽ được thể hiện 9/8/4.png đối với raster tiles, 9/8/4 geojson với vector tiles. Vector tiles cho phép các mức thu phóng tăng theo số thập phân (ví dụ 6,5) thay vì chỉ các số nguyên như raster tiles $6,7,8$. (Xem hinh 4)

Các định dạng vector tiles phổ biến gồm GeoJSON, TopoJSON và Mapbox Vector tiles (MVT). GeoJSON là nền tảng lưu trữ dữ liệu dạng điểm, đường, vùng, đa giác và tập hợp hình học (geometry collections), nó có cấu trúc đơn giản và độc lập với nền tảng sử dụng. TopoJSON là nền tảng topo mở rộng của GeoJSON không lưu các đối tượng hình học riêng biệt thành điểm, đường, vùng mà lưu dữ liệu theo kiểu 1 đối tượng chung, điều này giúp giảm $80 \%$ kích cỡ của dữ liệu. Mapbox vector tiles là định dạng dữ liệu mở dựa trên google Proto. Các tiles được tổ chức theo Google Schema sử dụng Web Mecator Coordiante System (ESPG:3857). Năm 2015 ESRI hỗ trợ định dạng này. Các đối tượng hình học được lưu trữ tương đối với gốc của mỗi ô lưới [2]

Thay đổi biểu tượng (symbol) các đối tượng trên bản đồ là ưu điểm lớn của vector tiles. Các máy khách có thể yêu cầu thay đổi kiểu dáng dữ liệu vector hiển thị trên bản đồ. Mỗi một kiểu dáng sẽ được tham chiếu tới dữ liệu được biểu thị theo một quy tắc. Một vài chuẩn định nghĩa làm sao một biểu tượng trên bản đồ được áp dụng như Mapbox GL Styles, CartoCSS, MapCSS, vv ...

\subsection{Sü dụng NodeJS tạo dịch vụ tiles server}

NodeJS hiện đang là một trong những framework rất phổ biến trong việc xây dựng các ứng dụng web [5]. Phần này của bài báo sẽ trình bày phương pháp sử dụng framework NodeJS để tạo ra một tiles server.

Để xây dựng dịch vụ tiles server trước tiên, ta cần lưu dữ liệu trọng một hệ quản trị cơ sở dữ liệu. Nghiên cứu này đề xuất dữ liệu bản đồ sẽ được lưu trong hệ quản trị cơ sở dữ liệu PostGreSQL [6]. PostgreSQL là hệ quản trị cơ sở dữ liệu mã nguồn mở miễn phí và tích hợp thành phần mở rộng PostGIS cho phép lưu trữ, truy vấn dữ liệu không gian một cách thuận tiện. Các dạng dữ liệu không gian phổ biện được sử dụng như GeoJSON hay shape file đều có thể dễ dàng đưa vào lưu trữ trong các bảng dữ liệu cửa

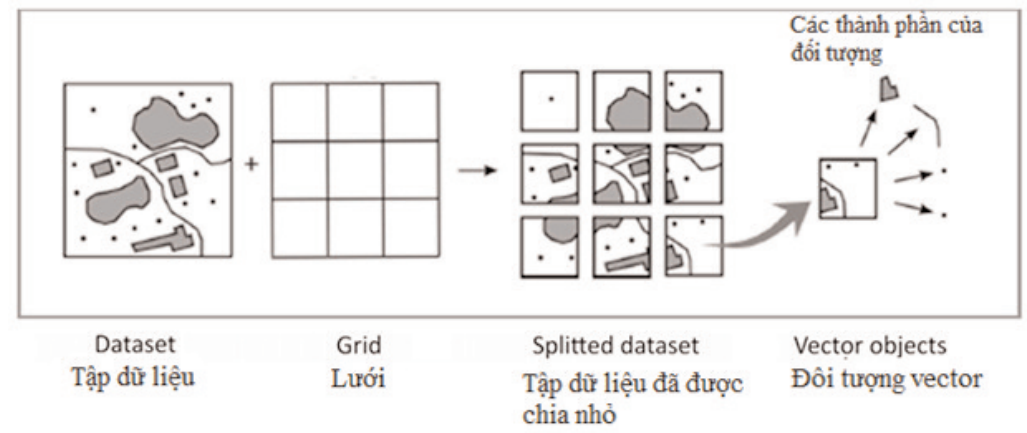

Hình 4: Cách thức tạo vector tiles 


\section{PostgreSQL.}

Để tạo ra một tile server, nghiên cứu đề xuất sử dụng framework Express [7] dành cho NodeJS. Framework Express giúp tạo các điều hướng (router trong hệ thống máy chủ) thông qua các tham số trên đường dẫn của trang web, ví dụ như thêm các tham số tọa độ $\mathrm{x}, \mathrm{y}$, mức độ thu phóng z. Việc truy vấn dữ liệu theo tọa độ được truyền theo tham số được thể hiện bằng đoạn mã nguồn dưới đây:

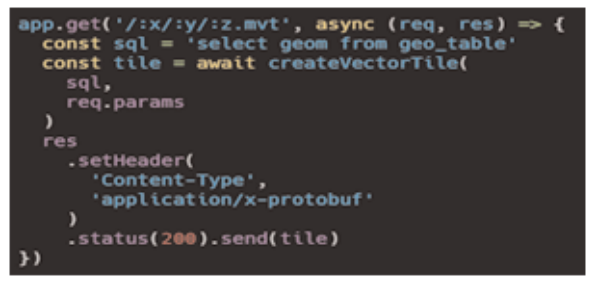

Việc điều hướng truyền tham số này sẽ được thực thi bằng câu lệnh $\mathrm{SQL}$ và sẽ trả về bằng ô lưới tương ứng với tọa độ $\mathrm{x}, \mathrm{y}$ và mức phóng $\mathrm{z}$.

Để tối ưu hóa khả năng tổng quát hóa bản đồ, nghiên cứu đề xuất sử dụng bộ thư viện MapNik [8]. Bộ thư viện này cho phép tối ưu hóa khả năng tổng quát hóa bản đồ ở các mức thu phóng khác nhau. Ví dụ dưới đây với mức thu phóng lớn sẽ thể hiện 3 đường thẳng, với mức thu phóng nhỏ hơn sẽ chỉ hiển thị còn một đường thẳng. Điều này giúp tăng khả năng hiển thị bản đồ một cách nhanh chóng.
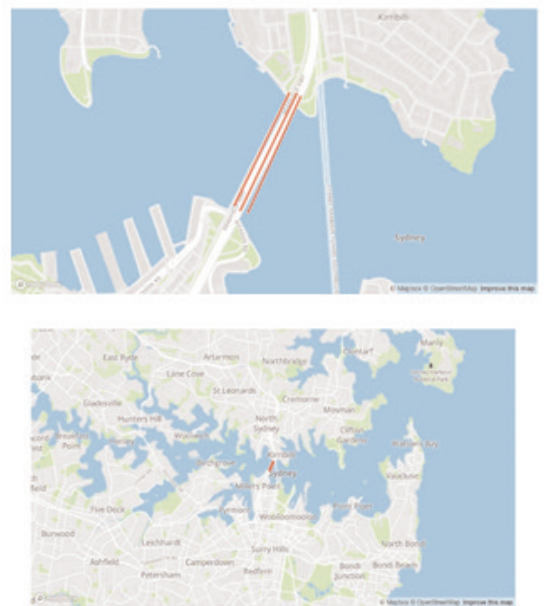

Hình 5: Vi dụ về khả năng tối ưu hóa hiển thị của thu viện MapNik
Để tạo một vector tiles, ta cần thực hiện bồn bước sau:

- Bước 1: định nghĩa hệ tọa độ

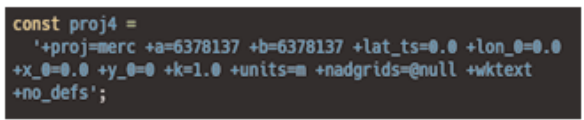

- Bước 2: thiết lập các thông số kết nối đến CSDL

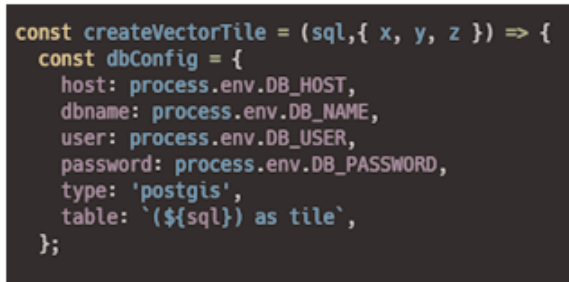

- Bước 3: Tạo các tiles

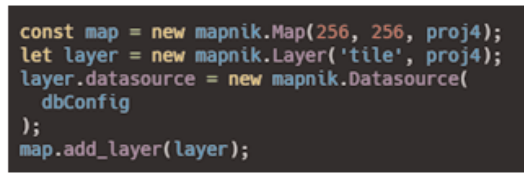

- Bước 4: Tạo vector tiles

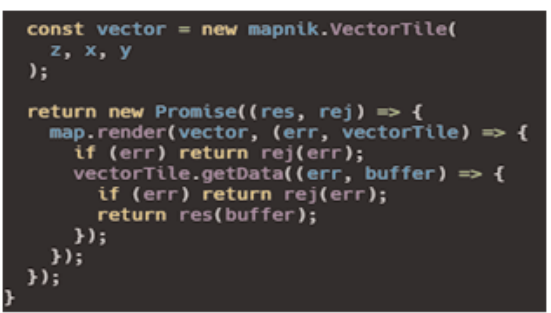

\section{Kết luận}

Kĩ thuật phân mảnh bản đồ (tiles map) hiện nay được các hệ thống lớn sử dụng như google map, bing map hay các hệ thống hiển thị ảnh vệ tinh. Tuy nhiên, tùy vào nhu cầu và đặc tính của các website khác nhau mà các nhà phát triển lựa chọn các dạng map tiles phù hợp. Ví dụ như đối với các trang web về ảnh vệ tinh không cần truy cập thông tin nhiều, không cần tùy biến về biểu tượng các đối tượng hiển thị trên ảnh, thì ta có thể sử dụng raster tiles. Hiện nay nhu cầu của người dùng các ứng dụng web phải cung cấp lượng lớn thông tin, bao gồm khả năng truy vấn thông tin theo yêu cầu, cũng như khả năng tùy biến các đối tượng trên bản đồ cho phù hợp với 
từng hoàn cảnh, mục đích. Do đó việc sử dụng định dạng Vector tiles gần như là bắt buộc và đang ngày trở nên phổ biến trên các ứng dụng web GIS. Bài báo đã trình bày tổng quan và phân tích ưu nhược điểm của cả hai dạng map tiles là raster tiles và vector tiles. Tiếp đó bài báo đã trình bày phương pháp xây dựng hệ thống cung cấp vector tiles đến người dùng bằng việc sử dụng NodeJS.

Đây là một phần kết quả nghiên cứu của đề tài "Hoàn thiện và chuyển giao mô hình tích hợp Thư viện điện tử Tây Nguyên phục vụ quản trị và truyền thông CSDL khoa học công nghệ", mã số: TN18/C05. Qua bài báo nhóm nghiên cứu gửi lời cảm ơn tới Ban Chương trình Khoa học và Công nghệ phục vụ phát triển kinh tế - xã hội vùng Tây Nguyên trong liên kết vùng hội nhập quốc tế 2018-2020 vì những định hướng và hỗ trợ quý báu trong suốt thời gian nghiên cứu. $\bigcirc$

\section{Tài liệu tham khảo}

[1]. Yilang Shen, Tinghua Ai, Jingzhong Li, Lu Wang, Wende Li, A tile-map-based method for the typification of artificial polygonal water areas considering the legibility, Computers \& Geosciences, Volume 143, 2020, 104552, ISSN 0098-3004,

https://doi.org/10.1016/j.cageo.2020.104552.

\section{Summary}

\section{Research on vector tiles and NodeJS framework for developing vector tiles services}

Nguyen Hoang Long, Đinh Bao Ngoc, Tran Thi Hai Van

\section{Hanoi University of Mining and Geology}

\section{Nguyen Dinh Ky, Lê Thị Kim Thoa}

Vietnam Academy Of science and technology

Optimizing the visualization and query function of web GISs needs to be considered when developing any web GIS application. Tiled map, especially raster tile has been provided by serveral GIS servers, such as ArcGIS server and GeoServer with the aim of increasing the visualization performance. Nevertheless, raster tiles could only provide data in the form of images. It leads to difficulties while querying data. Vector tile was proposed to improve those drawbacks of raster tiles. This paper presents the backgrounds of tiled map, including both raster tiles and vector tiles. The process of developing vector tiles services using an open sources software (PostgreSQL) and two frameworks (NodeJS and MapNik) is also introduced. $O$ 